\title{
Papers
}

\section{Effectiveness of targeted falls prevention programme in subacute hospital setting: randomised controlled trial}

\author{
Terry P Haines, Kim L Bennell, Richard H Osborne, Keith D Hill
}

\begin{abstract}
Objective To assess the effectiveness of a targeted, multiple intervention falls prevention programme in reducing falls and injuries related to falls in a subacute hospital.

Design Randomised controlled trial of a targeted multiple intervention programme implemented in addition to usual care compared with usual care alone.

Setting Three subacute wards in a metropolitan hospital specialising in rehabilitation and care of elderly patients. Participants 626 men and women aged 38 to 99 years (average 80 years) were recruited from consecutive admissions to subacute hospital wards.

Intervention Falls risk alert card with information brochure, exercise programme, education programme, and hip protectors.

Main outcome measures Incidence rate of falls, injuries related to falls, and proportion of participants who experienced one or more falls during their stay in hospital.

Results Participants in the intervention group $(n=310)$ experienced $30 \%$ fewer falls than participants in the control group $(\mathrm{n}=316)$. This difference was significant (Peto log rank test $\mathrm{P}=0.045$ ) and was most obvious after 45 days of

observation. In the intervention group there was a trend for a reduction in the proportion of participants who experienced falls (relative risk $0.78,95 \%$ confidence interval 0.56 to 1.06 ) and 28\% fewer falls resulted in injury (log rank test $\mathrm{P}=0.20)$. Conclusions A targeted multiple intervention falls prevention programme reduces the incidence of falls in the subacute hospital setting.
\end{abstract}

\section{Introduction}

Falls are a common occurrence among elderly inpatients in subacute hospitals and are generally reported to affect between 13\% and $32 \%$ of admitted patients. ${ }^{12}$ In stroke rehabilitation units, falls have been reported in up to $47 \%$ of patients. ${ }^{3}$ Patients who fall incur physical injuries (up to $70 \%$ of falls result in injuries, $1-10 \%$ result in fractures $),{ }^{45}$ psychological effects, ${ }^{6}$ and have longer lengths of hospital stay. ${ }^{5}$ Inpatient falls therefore result in substantial morbidity and additional healthcare costs and are a viable target for interventions.

Interventions to reduce in hospital falls have received little attention. Only three randomised controlled trials have been published. $^{7-9}$ Two investigated single interventions (bed alarms ${ }^{9}$ and alert bracelets ${ }^{8}$ ) in conjunction with usual care compared with usual care alone. The third investigated two interventions (additional exercise and type of flooring) in a two by two design in conjunction with usual care. ${ }^{7}$ None of these studies showed a significant reduction in fall rates, though all the studies were relatively small (between 54 and 134 participants). Previous studies based in hospitals with historical controls and randomised controlled trials in community settings have reduced fall rates by using targeted multiple intervention strategies. ${ }^{10}{ }^{11}$ We evaluated the effectiveness of a targeted multiple intervention falls prevention programme in reducing the rate of falls, the proportion of patients who fall, and the rate of injuries related to falls in a subacute hospital.

\section{Methods}

\section{Recruitment and randomisation}

All patients who were consecutively admitted to the Peter James Centre from March to December 2002 were eligible for inclusion. We approached family members or carers of patients for consent if the patient had communication difficulties or cognitive impairment (abbreviated mental test score on admission $<7$ ). There were no specific entry criteria other than referral from an acute hospital, accepted by a geriatrician. Patients ranged from 38 to 99 years, though most were over 70 years (average 80 ).

Members of the research team (physiotherapists or occupational therapists) approached patients as soon as practical after admission. We randomly allocated participants by using a random number table ${ }^{12}$ held at the centre by one investigator (TPH) who revealed allocation on receipt of written consent.

\section{Setting}

The Peter James Centre is a metropolitan hospital for rehabilitation and the care of elderly patients with three subacute wards (98 beds). There were no specific entry criteria other than referral from a geriatrician, most commonly from an acute hospital. Usual care entailed weekly medical assessments, one hour sessions of physiotherapy and occupational therapy each weekday, 24 hour nursing assistance, and other allied health services when required.

\section{Intervention}

Participants in the intervention group received a targeted falls prevention programme in addition to usual care. This programme consisted of a falls risk alert card with information brochure, an exercise programme, an education programme, and hip protectors (table 1). Hospital staff used their clinical judgment to determine the need and appropriateness of each of the interventions, after administration of the Peter James Centre falls risk assessment tool (PJC-FRAT, fig 1). This tool has an "admission sheet" for completion by nursing, physiotherapy, occupational therapy, and medical staff to guide them in recom- 


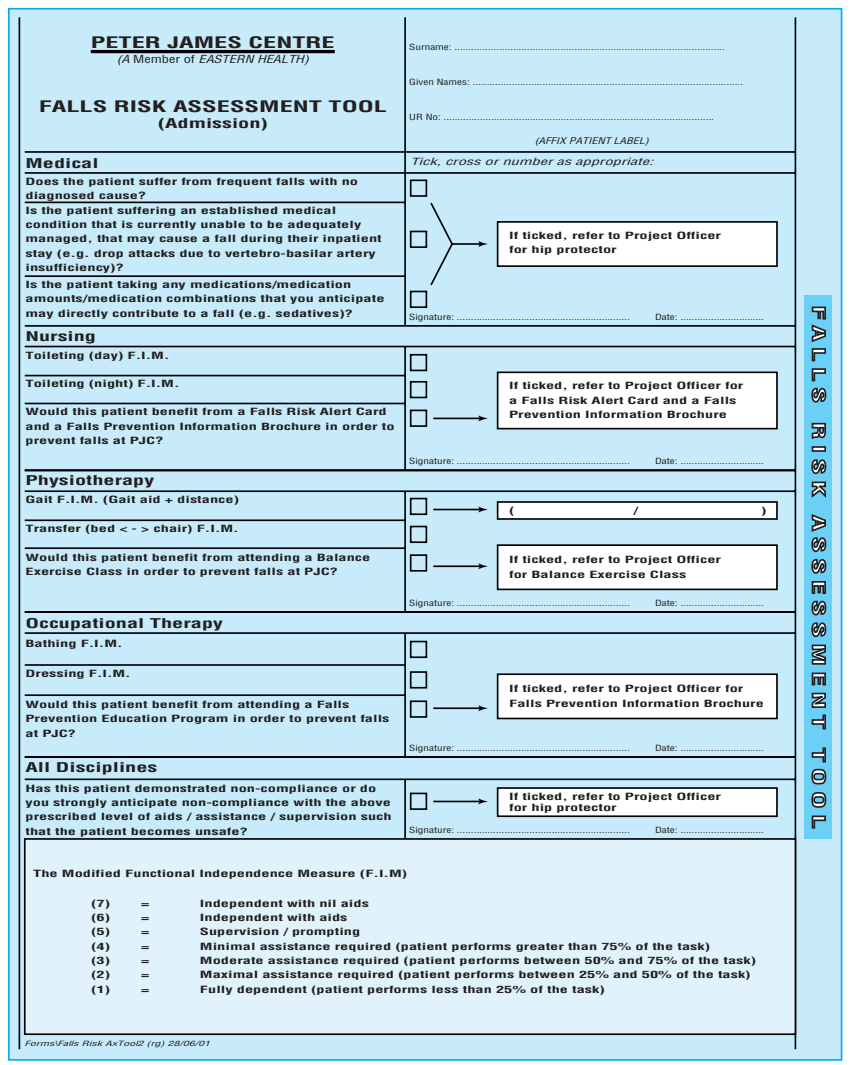

Fig 1 The Peter James Centre falls risk assessment tool (PJC-FRAT)

mending various combinations of the interventions (between none and all). The assessment tool also has an "amendment sheet," which staff could use to review their initial assessments on an "as required" basis.

The assessment tool was kept with the admission medical record of all patients, and hospital staff were asked to complete their section on the "admission sheet" as soon as possible after admission. Hospital staff were then asked to telephone research staff if they had recommended an intervention. Interventions were initiated within three days on receipt of recommendation, informed consent, and randomisation of the participant to the intervention group.

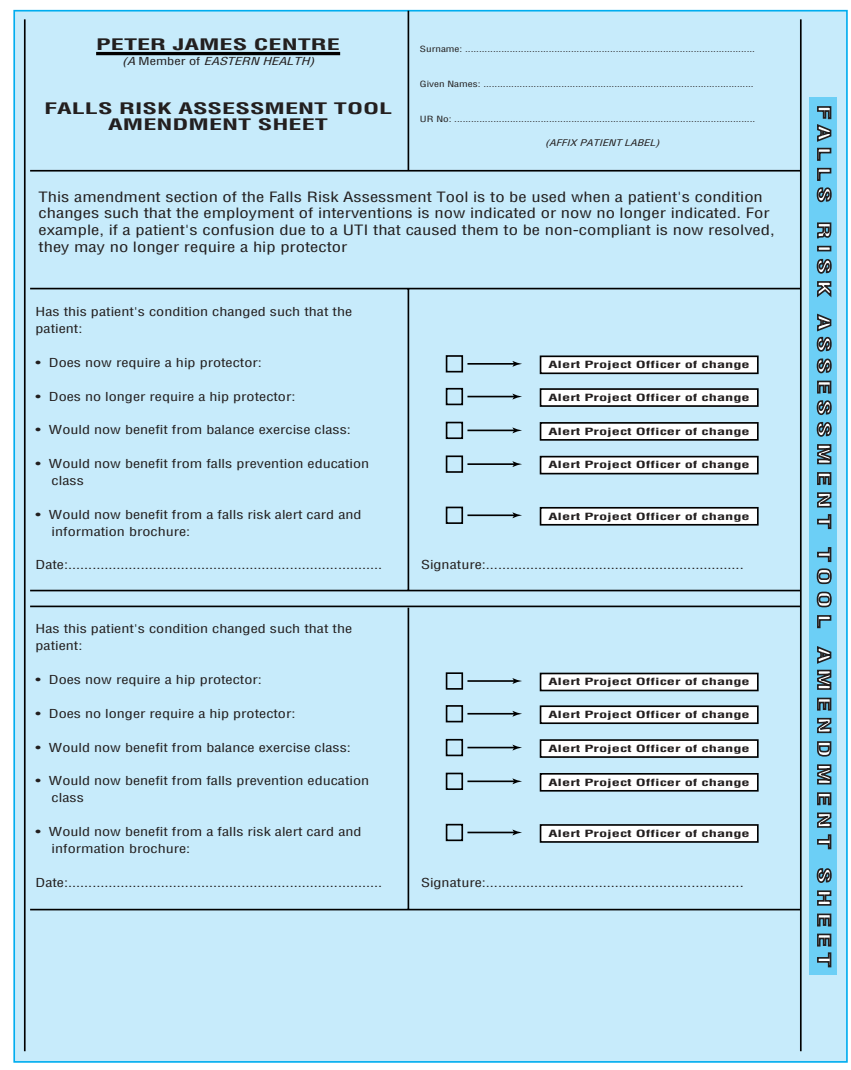

The PJC-FRAT was developed and refined in a prospective cohort of 122 consecutive admissions in which only usual care was provided. In this setting, the accuracy of the assessment tool for predicting fallers was acceptable. For example, figures for sensitivity and specificity for nursing staff were $73 \%$ and $75 \%$, respectively. These results, along with those for allied health and medical staff, were comparable with results from other screening instruments for risk of falls in this setting. ${ }^{13}$

Participants in the control group received usual care and did not receive any of the interventions from the falls prevention programme. Though hospital staff completed the assessment tool for these participants and generated recommendations for

Table 1 Four interventions and referring disciplines of targeted falls prevention programme

\begin{tabular}{|c|c|c|}
\hline Intervention & Description & Referring discipline \\
\hline Falls risk alert card & $\begin{array}{l}\text { Falls risk alert symbol on A4 size card placed above participant bed head. Information } \\
\text { brochure targeted at family members/carers to discuss with participant nature of hospital } \\
\text { falls and ways of preventing them }\end{array}$ & Nursing staff \\
\hline Exercise programme ${ }^{*}$ & $\begin{array}{l}\text { Three } 45 \text { minute sessions per week conducted by research physiotherapists in location } \\
\text { away from view of hospital physiotherapists. Exercises incorporated therapeutic principles } \\
\text { of t'ai chi }{ }^{22} \text { combined with functional activities such as transferring from chair to chair, } \\
\text { stepping, reaching, and weight shifting. Exercises tailored to meet individual abilities of } \\
\text { participants }\end{array}$ & Physiotherapy staff \\
\hline Education programme† & $\begin{array}{l}\text { Twice weekly individual sessions of up to } 30 \text { minutes conducted by research } \\
\text { occupational therapist at participants' bedside away from view of hospital occupational } \\
\text { therapists. Education manual that covered nature of hospital falls and how participants } \\
\text { can prevent them guided but did not limit content of these sessions. Programme } \\
\text { curriculum was covered over four sessions, though participants were allowed to receive } \\
\text { repetitions of education sessions as deemed appropriate }\end{array}$ & Occupational therapy staff \\
\hline Hip protectors & $\begin{array}{l}\text { Safehipł (force deflection—firm shield) hip protectors aimed at reducing incidence of } \\
\text { fractures of neck of femur. Hospital staff could decide part way into a participant's stay } \\
\text { that hip protector was no longer required }\end{array}$ & Medical, nursing, physiotherapy, and occupational therapy staff \\
\hline
\end{tabular}


intervention in the same manner as for participants in the intervention group, these interventions were not instituted (fig 2).

\section{Measurement of falls and related injuries}

Hospital staff generated a standardised incident report for all participants who fell during the study. Participants were promptly examined by hospital medical staff, who recorded any related injuries. We defined a fall as "any event when the participant unexpectedly came to rest on the ground, floor or another lower level."14 This definition was provided in writing to all hospital staff and discussed in preparation meetings before the study started. Participants were followed up until discharge from hospital or death.

\section{Data collection}

Hospital staff recorded baseline and outcome measures in medical histories by following standardised procedures. One investigator (TPH) collated these data after the participant was discharged.

Baseline measures included age, sex, living arrangements before admission to hospital, admission diagnostic category, ${ }^{15}$ medical history (stroke, Parkinson's disease, cancer, congestive heart failure, osteoporosis, or a fracture related to a fall), cognitive impairment (mini-mental state examination ${ }^{16}$ on admission), and functional dependency (modified Barthel index on admission).$^{15}$ We also recorded the level of compliance of hospital staff in completing the PJC-FRAT.

\section{Blinding}

The nature of the interventions prevented complete blinding of hospital staff and participants. In most cases, hospital staff who completed the PJC-FRAT "admission" sheet did not know to which group the patient had been allocated. The exception to this was in cases where the staff member was aware that a falls prevention programme intervention had been implemented based on another staff member's recommendations. Unblinding may have introduced several sources of bias, including altered practice in recording the incidence of falls or inconsistent provision of usual care. To gauge the level to which these biases may have been introduced, we conducted a survey of hospital staff (nursing, physiotherapy, and occupational therapy) eight months into the study period. Staff were asked to predict the group allocation of each participant they were caring for on that day.

\section{Statistical analysis}

We analysed primary outcomes on an intention to treat basis. We regarded data as recurrent events survival data, allowing all falls and variable participant study observation periods to be accounted for in the analysis. We compared the cumulative incidence of falls over time graphically using the Nelson-Aalen cumulative hazard estimator (a "step" function). ${ }^{17}$ Each participant's observation time started at time $=0$ (the $\mathrm{x}$ axis) regardless of when they were admitted to the hospital. The height of each "step" was determined by the number of falls within the group on one day (for example, day 10 since entering the study) divided by the number of participants in the group who had not yet been discharged (censored). We tested the equivalence of fall rates between groups using the $\log$ rank test. ${ }^{18}$ This test requires fall rates between groups to maintain a proportional relation over time. Some evidence suggests this may not have been the case (Schoenfeld residuals test ${ }^{19} \mathrm{P}=0.015$ ). Thus we also used the Peto extension of the $\log$ rank test as it adjusts for this non-proportionality. ${ }^{20}$

We compared the incidence of falls with injuries between groups using the log rank test. ${ }^{20}$ The proportion of participants

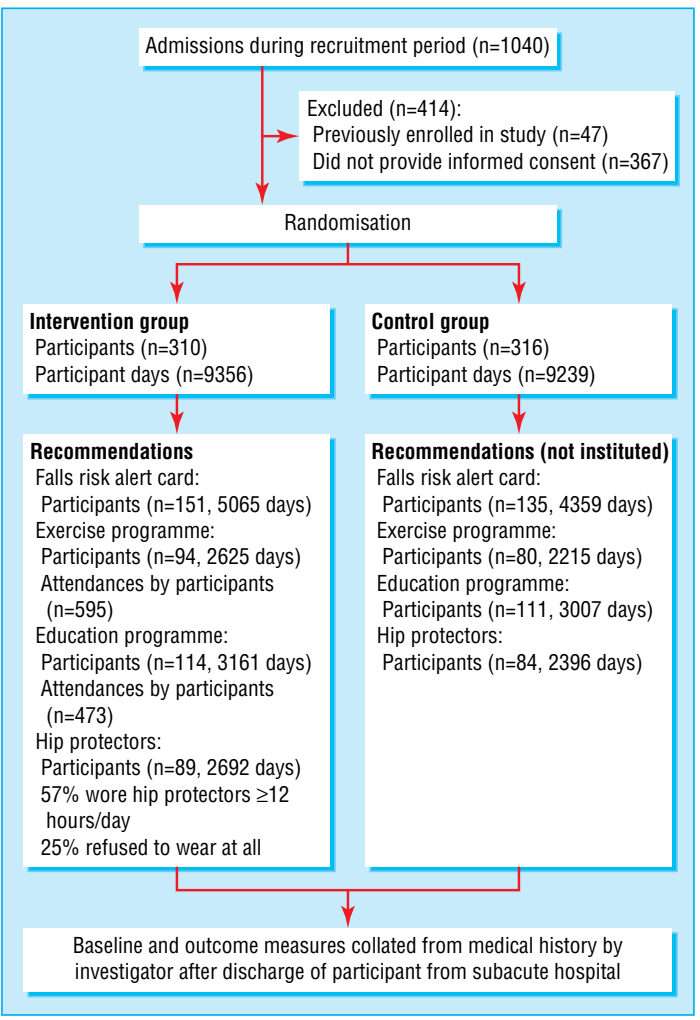

Fig 2 Recruitment, numbers allocated to control or intervention groups, and distribution of intervention subgroups

who experienced one or more falls was compared between groups using relative risk with $95 \%$ confidence intervals. ${ }^{21}$

The agreement between participant group allocation and hospital staff prediction of group allocation was analysed with the $\kappa$ statistic. $^{12}$ All analyses were conducted with Stata 8.0 software (StataCorp, College Station, TX).

\section{Sample size}

We calculated the sample size for this study by assuming that there would be 33\% fewer fallers in the intervention group compared with the control group. From hospital administrative data we projected that $30 \%$ of participants in the control group would fall. From these figures we then calculated that we would need 626 participants for a power of 0.80 , two tailed $\alpha=0.05 .^{12}$

\section{Results}

\section{Baseline characteristics}

We approached 1040 patients, of whom $626(60 \%)$ consented to participate. Figure 2 shows recruitment of participants and recommendations of the falls prevention programme intervention. No participants withdrew from the trial during the study period, and there were no adverse events attributable to the intervention. Baseline characteristics of participants in each group were similar (table 2).

\section{Analysis of falls, fallers, and injuries related to falls}

Compared with the control group, the intervention group had $30 \%$ fewer falls (149 $v 105)$ and a lower proportion of participants who experienced one or more falls (71 v54), relative risk $0.78(95 \%$ confidence interval 0.56 to 1.06$)$. Thirty five participants in the intervention group fell once compared with 49 in the control group. Both groups had 10 participants who fell twice and three who fell three times, but the intervention group 


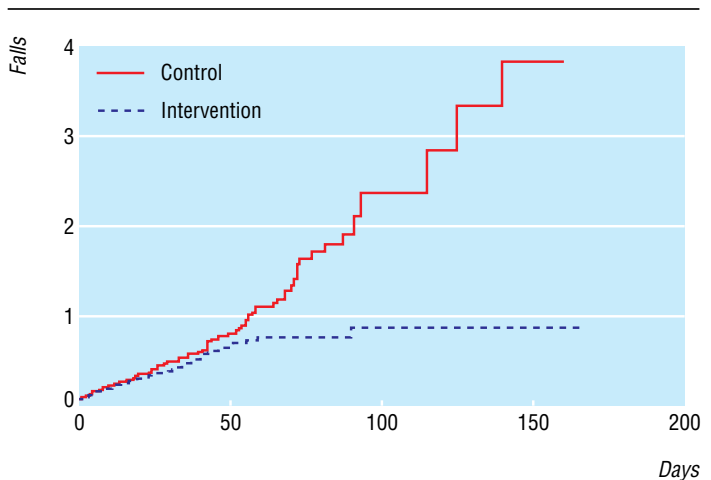

Fig 3 Nelson-Aalen cumulative hazard estimates of the control and intervention groups

had only six participants who fell four or more times compared with nine in the control group.

The Nelson-Aalen cumulative hazard estimate for both groups was similar until about day 45 , when the fall rate in the control group marginally increased and the rate in the intervention group suddenly reduced (fig 3). Both the log rank test $(\mathrm{P}=0.004)$ and Peto extension $(\mathrm{P}=0.045)$ showed significantly fewer falls in the intervention group.
Table 3 shows the definitions and distributions of injuries related to falls between the two groups. The incidence of falls with injury was $28 \%$ lower in the intervention group (23 v 32, log rank test, $\mathrm{P}=0.20$ ). Two participants from each group incurred a fracture related to a fall. One participant fractured the neck of the femur while wearing hip protectors.

\section{Hospital staff survey of participant group allocation}

Hospital staff correctly identified the group allocation of 90 out of 172 participants they were caring for $(\kappa=3 \%$ chance corrected agreement).

\section{Discussion}

This is the first large randomised trial to show that the incidence of falls in elderly patients in hospital can be significantly reduced. The targeted multiple intervention programme we used led to a $30 \%$ reduction. A reduction of this magnitude is important not only for individual patients and their families but also for hospital management in dealing with the associated costs and additional care needed because of falls. ${ }^{4-6}$ Falls prevention programmes in subacute hospitals have been based on the little evidence available from the hospital setting or by transferring results from other settings. This study provides valuable evidence for healthcare administrators and practitioners to reduce falls in

Table 2 Characteristics of participant and completion rates of the PJC-FRAT by hospital staff. Figures are numbers (percentages) unless stated otherwise

\begin{tabular}{|c|c|c|}
\hline Baseline & Control & Intervention \\
\hline Mean (SD) days from admission until consent & $2(2)$ & $2(3)$ \\
\hline Mean (SD) age (years) & $80(9)$ & $80(9)$ \\
\hline Men & $105(33)$ & $101(33)$ \\
\hline Modified Barthel index on admission $(/ 100)^{*}$ & $48(18)$ & $47(18)$ \\
\hline Admission mini-mental state examination $(30)^{\star} \dagger$ & $23(6)$ & $23(6)$ \\
\hline \multicolumn{3}{|l|}{ Diagnosis on primary admission: } \\
\hline Stroke & $40(13)$ & $32(10)$ \\
\hline Neurological & $12(4)$ & $13(4)$ \\
\hline Elective joint replacement & $44(14)$ & $33(11)$ \\
\hline Orthopaedic & $95(30)$ & $104(34)$ \\
\hline Other disabling impairment & $33(10)$ & $34(11)$ \\
\hline Other geriatric management & $53(17)$ & $57(18)$ \\
\hline \multicolumn{3}{|l|}{ Medical history: } \\
\hline Stroke & $70(22)$ & $65(21)$ \\
\hline Parkinson's disease & $18(6)$ & $18(6)$ \\
\hline Neoplasm & $49(16)$ & $45(15)$ \\
\hline Congestive cardiac failure & $40(13)$ & $49(16)$ \\
\hline Osteoporosis & $36(11)$ & $43(14)$ \\
\hline Fall related fracture & $55(17)$ & $68(22)$ \\
\hline \multicolumn{3}{|l|}{ Previous living: } \\
\hline Home alone & $120(38)$ & $118(38)$ \\
\hline Home with family & $143(45)$ & $144(46)$ \\
\hline Low level residential care facility & $52(16)$ & $47(15)$ \\
\hline Other & $1(0.3)$ & $1(0.3)$ \\
\hline Mean (SD) length of stay after consent (days) & $29(22)$ & $30(22)$ \\
\hline Mean (SD) No of attendances at hospital physiotherapy sessions & $22(17)$ & $23(20)$ \\
\hline \multicolumn{3}{|c|}{ Mean (SD) days from admission to completion of discipline specific PJC-FRAT section: } \\
\hline Medical & $3(5)$ & $4(5)$ \\
\hline Nursing & $1(7)$ & $1(2)$ \\
\hline Occupational therapy & $4(3)$ & $4(4)$ \\
\hline Physiotherapy & $1(2)$ & $1(2)$ \\
\hline \multicolumn{3}{|l|}{ Number of discipline specific PJC-FRAT sections not completed: } \\
\hline Medical & $138(43)$ & $112(36)$ \\
\hline Nursing & $32(10)$ & $27(9)$ \\
\hline Occupational therapy & $40(13)$ & $44(14)$ \\
\hline Physiotherapy & $14(4)$ & $6(2)$ \\
\hline
\end{tabular}

*Higher score

†25 missing values (13 control, 12 intervention) imputed by using best subset regression. 
Table 3 Classification and distribution of falls that led to injury (fall rated by worst injury sustained)

\begin{tabular}{lcc} 
Classification and description & Control & $2^{*}$ \\
\hline $\begin{array}{l}\text { Severe (fracture) } \\
\text { Moderate (laceration that required suturing, head injury requiring neurological observations to be taken, damage to } \\
\text { dentures, soft tissue injury requiring radiological investigations but no fracture) }\end{array}$ & 18 \\
\hline Minor (laceration that did not require suturing) & 12 \\
\hline Nil (no injuries were recorded) & 12 \\
\hline
\end{tabular}

${ }^{*}$ One neck of femur and one shaft of femur fracture.

†Two neck of femur fractures.

subacute hospitals, where falls are a common and dangerous occurrence.

In contrast to earlier studies, ${ }^{7-9}$ we have shown that this programme had a significant effect in reducing falls, which may be because of the targeted multiple intervention strategy, where each intervention intentionally addressed one or more of various risk factors for falls. There may also have been some unintended benefits, such as increased surveillance while participants were taking part in the exercise or education programmes. Targeting of interventions also meant that falls prevention strategies could be tailored to individual participants. The observed relative benefit of the intervention programme became pronounced after 45 days in hospital (fig 3), indicating that the programme may have had a cumulative effect over time and be of greatest benefit to those who have more complex presentations that necessitate greater lengths of stay in hospital.

Compared with the control group, 22\% fewer participants in the intervention group fell and there were $28 \%$ fewer falls with injuries. The proportion of participants who were fallers in the control group $(22.5 \%, 71$ fallers of 316 participants in group) was less than the projected proportion used for the power analysis (30\%). Our study was insufficiently powered to detect a difference in hip fractures (focus of the hip protector intervention) because they do not occur often (fractures have been reported to occur in less than $10 \%$ of hospital falls).

We focused on falls, fallers, and injury related to falls as separate end points. Examining falls is important as each individual fall potentially leads to negative outcomes (physical and psychological) for participants and places additional demands on hospital resources. Thus measuring a reduction in both falls and falls with physical injury indicates improved safety for participants and less drain on hospital resources due to falls. In examining fallers, participants are the unit of analysis rather than events (falls). A reduction in fallers indicates improved delivery of health care through a greater proportion of patients being treated without incurring falls and their potential consequences.

\section{Limitations and problems}

The inability to completely blind all staff and participants is a difficulty commonly encountered by researchers in the hospital setting. This may have influenced the recording of the incidence of falls or altered elements of usual care such as provision of regular physiotherapy. By randomising individual participants, variances between hospital wards in these recording behaviours should not have influenced the results. The staff blinding survey also indicated that hospital staff were relatively unaware of the allocation of participants. Lastly, attendances at the usual care physiotherapy sessions (table 2) were similar between groups, suggesting that the provision of usual care was unaffected.

Some ethical dilemmas were present in this study. Firstly, we approached family members or carers of participants with cognitive impairment to provide consent. Although this challenges autonomy, it is important to be able to recruit participants with cognitive impairment into research that may benefit this population. Participants were not forced to participate in any intervention and were free to withdraw from the study at any stage, thus preserving a large degree of participant autonomy. Secondly, though the falls risk alert card may violate participant privacy and cause distress to participants and their families, we used a falls alert symbol identifiable by hospital staff rather than a sign with words to minimise this risk. During the study no official complaints or requests to remove falls risk alert cards were received.

\section{Applying results}

The intervention programme could potentially be incorporated into the usual care of acute, other subacute, and residential facilities for elderly people. The principle underlying the PJC-FRAT - that hospital staff recommend falls prevention interventions based on their clinical judgment-can also be incorporated into these settings. Modifications to the exercise programme in the acute setting may be required to cater for participants with drips, drains, or other attachments. The description of the nature of falls provided in the written educational material could be modified and based on data on falls from local facilities.

Our results may be generalisable to other subacute settings. Although we recruited only $60 \%$ of eligible patients, their characteristics were consistent with those of the consecutive sample of 122 patients used in the validation of the PJC-FRAT, which suggests our sample was reasonably representative. Many participants in this study had diagnoses of dementia or stroke and were recommended for the falls prevention interventions, indicating that the programme could be implemented on wards that deal specifically with patients with these diagnoses. However, generalising the findings to acute hospitals may be problematic as the reduction in falls rates occurred after 45 days, a period after which few acute patients would still be in hospital.

In this study, usual care at the centre was compared with usual care plus the targeted falls prevention programme. Subsequently, we could not investigate many falls prevention interventions, such as review of sedative medication prescription, using this approach as such interventions were already incorporated into usual care. These interventions, along with evaluation of the relative effectiveness of individual interventions from this programme and the cost effectiveness of targeted multiple intervention strategies, are worthy of further investigation.

We thank the participants; Lyn Watson, for statistical consultation on survival analysis, and the staff of the Peter James Centre; Ileanne Au and Thuan Nguyen, research physiotherapists; Paula Anastasaglou, Lesley Allard, and Kathleen Ballard, research occupational therapists; Amanda Stoneham, research assistant; and the Peter James Centre falls prevention project steering committee.

Contributors: All authors contributed to statistical analysis, and data interpretation and wrote the paper. TPH directed the project, designed the falls risk alert card, falls prevention information brochure, and the education and exercise programmes, and performed duties of research physiotherapists and occupational therapists if necessary. TPH, KLB, and $\mathrm{KDH}$ contributed to the protocol design. TPH is guarantor.

Funding: Victorian Department of Human Services, Aged Care Division. 


\section{What is already known on this topic}

Although several randomised controlled trials in community settings support the use of targeted multiple intervention programmes in reducing falls, there is little evidence of their effectiveness in hospitals

The three published trials that investigated falls prevention interventions in the hospital setting were underpowered and did not show a significant reduction in falls

\section{What this study adds}

A targeted multiple intervention falls prevention programme in addition to usual care compared with usual care alone reduced the incidence rate of falls by $30 \%$ in a subacute hospital setting

There was also a trend towards a reduction in the proportion of participants who were "fallers" and the incidence rate of fall related injuries

A targeted programme in addition to usual care leads to safer stays in hospitals for patients

Competing interests: None declared.

Ethical approval: Human Research Ethics Committee of the Peter James Centre.

1 Mion L, Gregor S, Buettner M, Chwirchak D, Lee O, Paras W. Falls in the rehabilitation setting: incidence and characteristics. Rehabil Nurs 1989;14:17-22.

2 Vlahov D, Myers A, Al-Ibrahim M. Epidemiology of falls among patients in a rehabilitation hospital. Arch Phys Med Rehabil 1990;71:8-12.

3 Rapport LJ, Webster JS, Flemming KL, Lindberg JW, Godlewski MC, Brees JE, et al. Predictors of falls among right-hemisphere stroke patients in the rehabilitation setting. Arch Phys Med Rehabil 1993;74:621-6.

4 Brandis S. A collaborative occupational therapy and nursing approach to falls prevention in hospital inpatients. J Qual Clin Pract 1999;19:215-20.

5 Grenier-Sennelier C, Lombard I, Jeny-Loeper C, Maillet-Gouret M, Minvielle E. Designing adverse event prevention programs using quality management methods: the case of falls in hospital. Int J Qual Health Care 2002;14:419-26.
6 Liddle J, Gilleard C. The emotional consequences of falls for older people and their families. Clin Rehabil 1995;9:110-4.

Donald I, Shuttleworth H. Preventing falls on an elderly care rehabilitation ward. Clin Rehabil 2000;14:178-85.

Mayo N, Gloutney L, Levy R. A randomised trial of identification bracelets to preven falls among patients in a rehabilitation hospital. Arch Phys Med Rehabil 1994;75:1302-8.

9 Tideiksaar R, Feiner C, Maby J. Falls prevention: The efficacy of a bed alarm system in an acute care setting. Mount Sinai J Med 1993;60:522-7.

10 Oliver D, Hopper A, Seed P. Do hospital fall prevention programs work? A systematic review. J Am Geriatr Soc 2000;48:1679-89.

11 Gillespie L, Gillespie W, Robertson M, Lamb S, Cumming R, Rowe B. Interventions for preventing falls in elderly people. Cochrane Database Syst Rev 2003;(4):CD000340.

12 Freventing falls in elderly people. Cochrane Database Syst Rev 2003;(4):CD000340.

13 Perell K, Nelson A, Goldman R, Luther S, Prieto-Lewis N, Rubenstein L. Fall risk Perell K, Nelson A, Goldman R, Luther S, Prieto-Lewis N, Rubenstein L. Fall risk
assessment measures: an analytic review. J Gerontol A Biol Sci Med Sci 2001;56:M761-6. assessment measures: an analytic review. J Gerontol A Biol Sci Med Sci 2001;56:M761-6.
4 Wolf S, Barnhart H, Kutner N, McNeely E, Coogler C, Xu T, et al. Reducing frailty and falls in older persons: an investigation of tai chi and computerized balance training. JAm Geriatr Soc 1996;44:489-97.

15 Victorian Department of Human Services. Victorian admitted episode dataset. Melbourne: Victorian Department of Human Services-Australia, 2002.

16 Folstein M, Folstein S, McHugh P. Mini-mental state: A practical method for grading the cognitive state of patients for the clinician. J Psychiatr Res 1975;12:189-98.

17 Nelson W. Theory and applications of hazard plotting for censored failure data Technometrics 1972;14:945-65.

18 Pepe M, Cai J. Some graphical displays and marginal regression analyses for recurrent failure times and time dependent covariates. JAm Stat Assoc 1993;88:811-20.

19 Grambsch P, Therneau T. Proportional hazards tests and diagnostics based on weighted residuals. Biometrika 1994;81:515-26.

20 Peto R, Peto J. Asymptotically efficient rank invariant test procedures (with discussion). JR Stat Soc A 1972;135:185-206.

21 Altman D. Practical statistics for medical research. 1st ed. Melbourne: Chapman and Hall, 1993.

22 Wolf S, Kutner N, Green R, McNeely E. The Atlanta FICSIT study: Two exercise interventions to reduce frailty in elders. JAm Geriatr Soc 1993;41:329-32.

(Accepted 15 January 2004)

bmj.com 2004;328:676

University of Melbourne School of Physiotherapy, Parkville, Victoria 3052, Australia

Terry P Haines PhD candidate

Kim L Bennell associate professor

Centre for Rheumatic Disease, Royal Melbourne Hospital and the University of

Melbourne, Parkville

Richard H Osborne senior lecturer

National Ageing Research Institute and Melbourne Extended Care and

Rehabilitation Service, Parkville

Keith D Hill senior research fellow

Correspondence to: K Hill

k.hill@nari.unimelb.edu.au 